\title{
ON THE GENERATING FUNCTIONS OF MERSENNE AND FERMAT PRIMES
}

\author{
PABLO A. PANZONE
}

\begin{abstract}
With the aid of Solomon Golomb formula we give closed formulas for the generating functions of Mersenne and Fermat primes. A limit-type criteria for the existence of an infinite number of such primes is given using these generating functions.
\end{abstract}

\section{INTRODUCTION AND RESULTS.}

The arguments given to support conjectures about Mersenne and Fermat primes are, as far as the author knows, of probabilistic nature (see [2] pgs. 19-28, [3],[8],[9] and references therein).

The aim of this note is to give exact closed form formulas for the generating functions of Mersenne and Fermat primes using Solomon Golomb's formula [4].

We use this generating functions to give limit-type criteria for the existence of an infinite number of such primes. Our main results are Theorem 1 (generating functions for Mersenne and Fermat primes), Theorem 2 (criteria for existence of such primes). Proofs are given in Section 3.

S. Golomb proposed a general method in number theory for dealing with general conjectures. As an example he used his formula (1.2) to show in [4] a formal proof of the twin prime conjecture. His proof only lacked a powerful enough abelian theorem. Our results are discussed in Section 2 in this spirit with a tentative heuristic argument. In some sense Golomb's method may be viewed as a generalization of N. Wiener proof of the prime number theorem.

We denote the set of Mersenne and Fermat primes by $\mathbf{M}$ and $\mathbf{F}$ respectively, that is, primes of the form $2^{n}-1$ and $2^{n}+1$. Thus $\mathbf{M}=\{3,7,31,127, \ldots\}$ and $\mathbf{F}=\{3,5,17,257,65537, \ldots\}$. Many Mersenne primes are known but only five Fermat primes are known (those stated). Observe that the number 3 is both a Fermat and a Mersenne prime. The following two facts are well-known: if $4<2^{n}+1$ is prime then $n$ is a power of 2 and if $2^{n}-1$ is prime then $n$ is prime.

This note is self-contained and in Lemma 1 we give a slight generalization of Golomb's formula for a special case .

Our notation is as follows. For a natural number $m$ we write $m=p_{1}^{r_{1}} \cdots p_{\ell}^{r_{\ell}}$ where $p_{i}$ are distinct primes and $r_{i} \geq 1$ (we shall use always $p$ for a prime number). We denote by $\nu(m)$ a completely additive function i.e. a function defined at positive integer numbers such that $\nu(a)+\nu(b)=\nu(a b)$. From this one sees that $\nu(m)=r_{1} \nu\left(p_{1}\right)+\ldots+r_{\ell} \nu\left(p_{\ell}\right), \nu(1)=0$. At the other hand, any function $f$ defined only at prime numbers may be extended to give a (unique) completely additive function defining $\nu(1)=0$ and $\nu(m)=r_{1} f\left(p_{1}\right)+\ldots+r_{\ell} f\left(p_{\ell}\right)$. We write as usual

Research supported in part by Conicet. 
$\omega(m)=\ell$ and $\zeta(s), \mu(d), \Lambda(d)$ stand for the Riemann-Zeta function, the Möbius function and the von Mangold function respectively (see [7]). Finally we recall the definition of theta functions (see [1]),

$$
\begin{gathered}
\theta_{3}(q)=\sum_{n=-\infty}^{\infty} q^{n^{2}}, \quad \theta_{4}(q)=\theta_{3}(-q), \\
\theta_{2}(q)=\sum_{n=-\infty}^{\infty} q^{(n+1 / 2)^{2}} .
\end{gathered}
$$

The following is a slight generalization of Golomb's formula.

Lemma 1. Let $g(n, m)$ be any complex or real function of the positive integers $n, m$ and $\nu(d)$ any completely additive function. Then

$$
\begin{gathered}
\sum_{d \mid m=p_{1}^{r_{1}} \ldots p_{\ell}^{r_{\ell}}} \mu(d) \nu^{2}(d) g(\omega(d), m)=\left\{\sum_{i=1}^{\ell} \nu^{2}\left(p_{i}\right)\right\}\left\{\sum_{j=0}^{\ell-1}(-1)^{j+1}\left(\begin{array}{c}
\ell-1 \\
j
\end{array}\right) g(j+1, m)\right\}+ \\
2\left\{\sum_{1 \leq i<j \leq \ell} \nu\left(p_{i}\right) \nu\left(p_{j}\right)\right\}\left\{\sum_{j=0}^{\ell-2}(-1)^{j}\left(\begin{array}{c}
\ell-2 \\
j
\end{array}\right) g(j+2, m)\right\} .
\end{gathered}
$$

Example. If we set $g(n, m)=x^{n}$ and $\nu(d)$ any completely additive function then we get

$$
\begin{gathered}
\sum_{d \mid m=p_{1}^{r_{1}} \ldots p_{\ell}^{r_{\ell}}} \mu(d) \nu^{2}(d) x^{\omega(d)}= \\
=\left\{\begin{array}{l}
-x \nu^{2}\left(p_{1}\right), \text { if } \ell=1 \\
-x(1-x)\left(\nu^{2}\left(p_{1}\right)+\nu^{2}\left(p_{2}\right)\right)+x^{2} 2 \nu\left(p_{1}\right) \nu\left(p_{2}\right), \text { if } \ell=2 \\
-x(1-x)^{\ell-1}\left(\sum_{1 \leq i \leq \ell} \nu^{2}\left(p_{i}\right)\right)+x^{2}(1-x)^{\ell-2} 2\left(\sum_{i<j} \nu\left(p_{i}\right) \nu\left(p_{j}\right)\right), \text { if } \ell>2 .
\end{array}\right.
\end{gathered}
$$

If one puts $x=1$ one gets Golomb's formula which is not zero only when $\ell=$ 1 or 2 . Indeed one has

$$
\sum_{d \mid m=p_{1}^{r_{1} \ldots} p_{\ell}^{r_{\ell}}} \mu(d) \nu^{2}(d)=\left\{\begin{array}{l}
-\nu^{2}\left(p_{1}\right), \text { if } \ell=1 \\
2 \nu\left(p_{1}\right) \nu\left(p_{2}\right), \text { if } \ell=2 \\
0, \text { if } \ell>2 .
\end{array}\right.
$$

Using this, one proves the following key lemma:

Lemma 2. Let $m=n^{2}-1$, with nodd $>1$. Set $a_{m}=\sum_{d \mid m} \mu(d) \nu^{2}(d)$, where $\nu(d)$ is any completely additive function. Then $a_{m}$ is zero unless the following is true:

i) $m=8$ and then $a_{8}=-\nu^{2}(2)$.

ii) $m=(2 p+1)^{2}-1$ where $p$ is a Mersenne prime and then $a_{m}=2 \nu(2) \nu(p)$.

iii) $m=(2 p-1)^{2}-1$ where $p$ is a Fermat prime and then $a_{m}=2 \nu(2) \nu(p)$.

iv) $m=288=2^{5} 3^{2}=n^{2}-1=17^{2}-1$ and then $a_{288}=2 \nu(2) \nu(3)$. 
Recall that we denote the set of Mersenne and Fermat primes by $\mathbf{M}$ and $\mathbf{F}$ respectively. Using Lemma 2 one can prove the following closed form formulas (we warn the reader that there is some clash of notation for the variable $z$ used and theta functions)

Theorem 1. i) Let $\nu(d)$ be any completely additive function such that $\nu(d)=$ $O\left(d^{k}\right)$ for some $0<k$. If $|z|<1$ then

$$
\begin{gathered}
\nu(3) z^{17}+\sum_{p \in \mathbf{M}} \nu(p) z^{2 p+1}+\sum_{p \in \mathbf{F}} \nu(p) z^{2 p-1}= \\
=-\sum_{d=3 ; \text { odd }}^{\infty} \mu(d) \frac{\nu(d)}{1-z^{2 d}}\left\{\sum_{j=1}^{2^{\omega(d)}} z^{a_{j}}\right\},
\end{gathered}
$$

where $1<a_{1}<a_{2}<a_{3}<\ldots<a_{2^{\omega(d)}-1}<2 d, a_{2^{\omega(d)}}=2 d+1$ are a complete system of odd solutions of the congruence $a^{2}=1 \operatorname{modd}(d$ odd $)$.

ii) The same hypothesis as in i). If $\chi$ is the non principal Dirichlet character mod 4 , that is $\chi(1)=1, \chi(2)=0, \chi(3)=-1, \chi(4)=0$, and $|z|<1$, then

$$
\begin{gathered}
-\nu(3) z^{17}+\sum_{p \in \mathbf{M}} \nu(p) z^{2 p+1}-\sum_{p \in \mathbf{F}} \nu(p) z^{2 p-1}= \\
=\sum_{d=3 ; d o d d}^{\infty} \mu(d) \frac{\nu(d)}{1+z^{2 d}}\left\{\sum_{j=1}^{2^{\omega(d)}} \chi\left(a_{j}\right) z^{a_{j}}\right\} .
\end{gathered}
$$

iii) Let $r$ be a fixed real number such that $0<t<r<1$ and $\gamma_{r}$ an anticlockwise circle of radius $r$ centered at zero. Set $H(w):=\sum_{m=1}^{\infty} h(m) w^{m} ; \sum_{m=1}^{\infty} \frac{h(m)}{m^{s}}:=$ $-\left(\frac{\zeta^{\prime}(s)}{\zeta(s)}\right)^{\prime}+\left(\frac{\zeta^{\prime}(s)}{\zeta(s)}\right)^{2}$ and $G(q):=\frac{\theta_{3}(q)-\theta_{4}(q)}{4 q}-1$.

$$
\begin{gathered}
-\log ^{2} 2 t^{8}+2 \log 2 \log 3 t^{288}+2 \log 2 \sum_{p \in \mathbf{M}} \log p t^{4 p(p+1)}+2 \log 2 \sum_{p \in \mathbf{F}} \log p t^{4 p(p-1)} \\
=\frac{1}{2 \pi i} \int_{\gamma_{r}} H(q) G\left(\frac{t}{q}\right) \frac{d q}{q}=\frac{1}{2 \pi i} \int_{\gamma_{r}} G(q) H\left(\frac{t}{q}\right) \frac{d q}{q}
\end{gathered}
$$

iv) With the same notation as in i). Let $\chi$ be the non principal Dirichlet character $\bmod 4$. If $d=p_{1}^{r_{1}} \cdots p_{\ell}^{r_{\ell}},|z|<1$, then

$$
2 \sum_{3<p \in \mathbf{F}} z^{2 p-1}=-\sum_{d=3 ; d o d d}^{\infty} \mu(d)\left\{\frac{\omega(d)+\sum_{i=1}^{\omega(d)} \chi\left(p_{i}\right)}{1-z^{2 d}}\right\}\left\{\sum_{j=1}^{2^{\omega(d)}} z^{a_{j}}\right\} .
$$

Remarks: Notice that in (1.3), (1.4) or (1.6)

$$
a_{1}+a_{2^{\omega(d)}-2}=a_{2}+a_{2^{\omega(d)}-3}=a_{3}+a_{2^{\omega(d)}-4}=\ldots=2 d,
$$




$$
\begin{gathered}
a_{2^{\omega(d)}-1}=2 d-1, \\
\sqrt{2 d+1} \leq a_{1} .
\end{gathered}
$$

This last inequality shows, for example, that $\sum_{d=3 ; d o d d}^{\infty}\left|\mu(d) \frac{\nu(d)}{1-z^{2 d}}\left\{\sum_{j=1}^{2^{\omega(d)}} z^{a_{j}}\right\}\right|$ is convergent for $|z|<1$ (at least). The same holds for the series in (1.4) and (1.6). Of course one may add or subtract formulas (1.3) and (1.4) to give other closed form formulas for Mersenne or Fermat primes alone.

Also we notice that in (1.5) the function $G(q)$ transforms like ([1] pg. 40)

$$
G\left(e^{-y \pi}\right)=\frac{\theta_{3}\left(e^{-y \pi}\right)-\theta_{4}\left(e^{-y \pi}\right)}{4 e^{-y \pi}}-1=\frac{\theta_{3}\left(e^{-\pi / y}\right)-\theta_{2}\left(e^{-\pi / y}\right)}{4 \sqrt{y} e^{-y \pi}}-1 .
$$

Using Theorem 1 one can obtain the following criteria:

Theorem 2 (Criteria for Mersenne-Fermat primes). Let $\nu(d)$ be any completely additive function such that $\nu(d)=O\left(d^{k}\right)$ for some $0<k$. Also let $1<a_{1}<a_{2}<a_{3}<\ldots<a_{2^{\omega(d)}-1}<2 d, a_{2^{\omega(d)}}=2 d+1$ be a complete system of odd solutions of the congruence $a^{2}=1 \bmod d$ ( $d$ odd).

Assume that it is not true that

$$
\lim _{z \rightarrow 1-} \sum_{d=3 ; d o d d}^{\infty} \frac{\mu(d) \nu(d)}{1+z+z^{2}+\ldots+z^{2 d-1}}\left\{\sum_{j=1}^{2^{\omega(d)}} z^{a_{j}}\right\}=0 .
$$

Then $\boldsymbol{M} \bigcup \boldsymbol{F}$ is infinite.

\section{Discussion.}

Our first observation is that if $\nu(p)=1$ then formula (1.8) is indeed true: clearly $\sum_{p} z^{2 p}=O\left(\sum_{l \geq 1} z^{2^{l}}\right)$ when $p$ runs through all the Mersenne and Fermat primes. Now, noting that $\sum_{l \geq 1} z^{2^{l}} \leq m-1+\sum_{l \geq 0} z^{2^{m} l}=m-1+\frac{1}{1-z^{2^{m}}}$ for any $1 \leq m$, we see that

$$
\lim _{z \rightarrow 1-}(1-z)\left|z^{17}+\sum_{p \in \mathbf{M}} z^{2 p+1}+\sum_{p \in \mathbf{F}} z^{2 p-1}\right| \leq C \lim _{z \rightarrow 1-}(1-z) \frac{1}{1-z^{2^{m}}}=\frac{C}{2^{m}} .
$$

The result follows by making $m \rightarrow \infty$ and using Theorem $1 \mathrm{i}$ ).

Next it is interesting to note that if $\nu(p)=p$ one expects the limit of (1.8) to be non zero. We discuss this from a formal viewpoint. Formal here means to take the limit termwise in formula (1.8) (or other formulas) and evaluate the resulting series.

We give now a non rigorous argument showing that the resulting series in (1.8) is not zero. Indeed the left hand side of $(1.8)$, with the choice $\nu(p)=p$, is equal to 


$$
\lim _{z \rightarrow 1-} \sum_{d=3 ; d \text { odd }}^{\infty} \frac{\mu(d) \nu(d)\left\{\sum_{j=1}^{2^{\omega(d)}} z^{a_{j}}\right\}}{1+z+\ldots+z^{2 d-1}} \cong \sum_{d=3 ; d o d d ; d=p_{1} \cdots p_{\ell}}^{\infty} \frac{\mu(d) 2^{\omega(d)}\left(p_{1}+\ldots+p_{\ell}\right)}{2 d}
$$

where $p_{i} \neq p_{j}$ and we have written $\cong$ meaning a formal equality.

Define function $P(t, x, s)$ for $0<s, x ; 0<t<1$, as follows:

$$
P(t, x, s):=\frac{1}{2} \Pi_{2<p, \text { prime } \leq x}\left(1-\frac{2 t^{p}}{p^{s}}\right)=\frac{1}{2} \sum \frac{\mu(d) 2^{\omega(d)} t^{p_{1}+\ldots+p_{\ell}}}{d^{s}},
$$

where $\sum^{\prime}$ indicates a certain finite sum over $d=p_{1} \cdots p_{\ell}$. As we shall see, the following lemma holds.

\section{Lemma 3.}

$$
\lim _{t \rightarrow 1-, x \rightarrow \infty} \frac{\partial P(t, x, 1)}{\partial t}=\infty
$$

(The double limit here means: for any integer $N$ there exists $\delta>0$ such that $\left|\frac{\partial P(t, x, 1)}{\partial t}\right|>N$ if $1-\delta<t<1$ and $\left.x>\frac{1}{\delta}\right)$.

But one has formally

$$
\begin{gathered}
\lim _{t \rightarrow 1-, x \rightarrow \infty} \frac{\partial P(t, x, 1)}{\partial t}=\lim _{t \rightarrow 1-, x \rightarrow \infty} \frac{1}{2} \sum \frac{\mu(d) 2^{\omega(d)}\left(p_{1}+\ldots+p_{\ell}\right) t^{p_{1}+\ldots+p_{\ell}}}{d} \cong \\
\sum_{d=3 ; d \text { odd; } ;=p_{1} \ldots p_{\ell}}^{\infty} \frac{\mu(d) 2^{\omega(d)}\left(p_{1}+\ldots+p_{\ell}\right)}{2 d} .
\end{gathered}
$$

Therefore this non rigorous argument gives that, with the choice $\nu(p)=p$, one has

$$
\lim _{z \rightarrow 1-} \sum_{d=3 ; d \text { odd }}^{\infty} \frac{\mu(d) \nu(d)\left\{\sum_{j=1}^{2^{\omega(d)}} z^{a_{j}}\right\}}{1+z+\ldots+z^{2 d-1}}=\infty
$$

Proof of Lemma 3: Let $\sum^{\prime \prime}=\sum_{2<p, \text { prime } \leq x}$, then

$$
\begin{gathered}
-\frac{\partial P(t, x, 1)}{\partial t}= \\
\exp \left\{\sum^{\prime \prime} \log \left(1-\frac{2 t^{p}}{p}\right)+\sum{ }^{\prime \prime} \frac{2 t^{p}}{p}\right\} \exp \left\{-2 \sum{ }^{\prime \prime} \frac{t^{p}}{p}\right\}\left(\sum^{\prime \prime} \frac{t^{p-1}}{1-\frac{2 t^{p}}{p}}\right)= \\
A(t, x) B(t, x) C(t, x) .
\end{gathered}
$$

Now $\lim _{t \rightarrow 1-, x \rightarrow \infty} A(t, x)=\exp \left(\sum_{p>2}^{\infty} \log \left(1-\frac{2}{p}\right)+\frac{2}{p}\right) \neq 0$.

Next assume $3 \leq x, 1 / 2<t<1$. Then $C(t, x) \geq \sum^{\prime \prime} t^{p}$ and using this, one has

$$
B(t, x) C(t, x) \gg \frac{m}{\log ^{3} m}, m:=\min \left\{x, \frac{1}{1-t}\right\} .
$$

In fact, this follows from $\sum^{\prime \prime} \frac{t^{p}}{p} \ll \log \log x, \sum^{\prime \prime} t^{p} \gg \frac{x}{\log x}$ if $x \leq \frac{1}{1-t}$ and $\sum^{\prime \prime} \frac{t^{p}}{p}=$ $\sum_{2<p \leq \frac{1}{1-t}} \frac{t^{p}}{p}+\sum_{\frac{1}{1-t}<p \leq x} \frac{t^{p}}{p} \ll \log \log \left(\frac{1}{1-t}\right)+O(1), \sum^{\prime \prime} t^{p} \geq \sum_{2<p \leq \frac{1}{1-t}} t^{p} \gg \frac{\frac{1}{1-t}}{\log \frac{1}{1-t}}$ 
if $x>\frac{1}{1-t}$. These inequalities are obtained using the prime number theorem and Mertens formula $\sum_{p \leq t_{0}} \frac{1}{p}=\log \log t_{0}+O(1)$.

\section{Proofs.}

Proof of Lemma 1: Set $m=p_{1}^{r_{1}} \cdots p_{\ell}^{r_{\ell}}$ and $m^{\prime}=p_{1} \cdots p_{\ell}$. Then

$$
\sum_{d \mid m} \mu(d) \nu^{2}(d) g(\omega(d), m)=\sum_{d \mid m^{\prime}} \mu(d) \nu^{2}(d) g(\omega(d), m)
$$

and using the fact $\nu\left(d^{\prime}\right)^{2}=\left(\sum_{p \mid d^{\prime}} \nu(p)\right)^{2}=\sum_{p \mid d^{\prime}} \nu(p)^{2}+2 \sum_{p q \mid d^{\prime}} \nu(p) \nu(q)$ if $d^{\prime} \mid m^{\prime}$ one gets

$$
\begin{gathered}
\sum_{d \mid m^{\prime}} \mu(d) \nu^{2}(d) g(\omega(d), m)= \\
-\sum_{p \mid m^{\prime}} \nu(p)^{2} \sum_{d \mid m^{\prime} / p} \mu(d) g(\omega(d)+1, m)+2 \sum_{p q \mid m^{\prime}} \nu(p) \nu(q) \sum_{d \mid m^{\prime} / p q} \mu(d) g(\omega(d)+2, m)
\end{gathered}
$$

and the result follows.

Proof of Lemma 2: Let $m=n^{2}-1$. Since $n$ is odd, $(n-1, n+1)=2$ and then, in order to make $\omega(m) \leq 2$, we must have either $n=2^{k}+1$ and $n+1=2 q^{a}$, or $n=2^{k}-1$ and $n-1=2 p^{b}$, for some $p$ and $q$ prime number and any positive integers $k, a, b$. In the first case we end up with the equation $2^{k-1}+1=q^{a}$ which, by the proof of Catalan's conjecture, is not possible unless $a=2, q=3, k=4$, which is (iv), $k=1$ which is (i) or $a=1$ and $q$ a Fermat prime which gives (iii). In the second case, we have $2^{k-1}-1=p^{b}$ which again is not possible unless $b=1$ and $p$ is a Mersenne prime, which is (ii). The result follows.

Proof of Theorem 1: i) It is an immediate consequence of Lemma 2 that

$$
\begin{gathered}
-\nu^{2}(2) z^{3}+2 \nu(2) \nu(3) z^{17}+2 \nu(2) \sum_{p \in \mathbf{M}} \nu(p) z^{2 p+1}+2 \nu(2) \sum_{p \in \mathbf{F}} \nu(p) z^{2 p-1}= \\
\sum_{n=3, n \text { odd }}^{\infty} z^{n}\left\{\sum_{d \mid n^{2}-1} \mu(d) \nu^{2}(d)\right\}=\sum_{d=1}^{\infty} \mu(d) \nu^{2}(d)\left\{\sum_{a^{2} \equiv 1(\text { mod } d) ; \text { o odd } \geq 3} z^{a}\right\}= \\
\sum_{d=1 ; \text { odd }}^{\infty} \mu(d) \nu^{2}(d)\left\{\sum_{a^{2} \equiv 1(\bmod d) ; \text { o odd } \geq 3} z^{a}\right\}+\sum_{d^{\prime}=1 ; d^{\prime} \text { even }}^{\infty} \mu\left(d^{\prime}\right) \nu^{2}\left(d^{\prime}\right)\left\{\sum_{a^{2} \equiv 1\left(\bmod d^{\prime}\right) ; \text { a odd } \geq 3} z^{a}\right\} \\
=\sum_{\text {odd }}+\sum_{\text {even }} .
\end{gathered}
$$

But $($ recall $\nu(1)=0)$

$$
\sum_{\text {odd }}=\sum_{d=3 ; d o d d}^{\infty} \frac{\mu(d) \nu^{2}(d)}{1-z^{2 d}}\left\{\sum_{j=1}^{2^{\omega(d)}} z^{a_{j}}\right\}
$$


where $1<a_{1}<a_{2}<a_{3}<\ldots<a_{2^{\omega(d)}-1}<2 d, a_{2 \omega(d)}=2 d+1$ are the odd solutions of the congruence $a^{2} \equiv 1 \bmod d$ ( $d$ odd).

Also, putting $d^{\prime}=2 d$ in the $\sum_{\text {even }}$ sum, one gets

$$
\begin{gathered}
\sum_{\text {even }}=\sum_{d=1 ; d \text { odd }}^{\infty} \mu(2 d) \nu^{2}(2 d)\left\{\sum_{a^{2} \equiv 1(\bmod 2 d) ; \text { a odd } \geq 3} z^{a}\right\}= \\
-\nu^{2}(2) \frac{z^{3}}{1-z^{2}}+\sum_{d=3 ; d \text { odd }}^{\infty} \mu(2 d) \nu^{2}(2 d)\left\{\sum_{a^{2} \equiv 1(\bmod 2 d) ; \text { o odd } \geq 3} z^{a}\right\}= \\
-\nu^{2}(2) \frac{z^{3}}{1-z^{2}}+\sum_{d=3 ; d \text { odd }}^{\infty} \frac{\mu(2 d) \nu^{2}(2 d)}{1-z^{2 d}}\left\{\sum_{j=1}^{2^{\omega(d)}} z^{a_{j}}\right\} .
\end{gathered}
$$

Notice in this last formula that $\mu(2 d) \nu^{2}(2 d)=-\mu(d)(\nu(2)+\nu(d))^{2}$. Taking into account this equality, add the $\sum_{\text {even }}$ sum and the $\sum_{\text {odd }}$ sum to obtain the following result:

Let $\nu(d)$ be any completely additive function such that $\nu(d)=O\left(d^{k}\right)$. Then if $|z|<1$

$$
\begin{gathered}
-\nu^{2}(2) z^{3}+2 \nu(2) \nu(3) z^{17}+2 \nu(2) \sum_{p \in \mathbf{M}} \nu(p) z^{2 p+1}+2 \nu(2) \sum_{p \in \mathbf{F}} \nu(p) z^{2 p-1}= \\
=-\nu^{2}(2) \frac{z^{3}}{1-z^{2}}-\nu(2) \sum_{d=3 ; \text { odd }}^{\infty} \mu(d) \frac{\{2 \nu(d)+\nu(2)\}}{1-z^{2 d}}\left\{\sum_{j=1}^{2^{\omega(d)}} z^{a_{j}}\right\} .
\end{gathered}
$$

Take in (3.1) the completely additive function $\nu$ defined by $f(p)=0$ for any prime $p>2, f(2)$ not zero and extended in the usual way as explained in the introduction. This yields

$$
-\nu(2)^{2} z^{3}=-\nu^{2}(2) \frac{z^{3}}{1-z^{2}}-\nu(2) \sum_{d=3 ; d o d d}^{\infty} \mu(d) \frac{\{\nu(2)\}}{1-z^{2 d}}\left\{\sum_{j=1}^{2^{\omega(d)}} z^{a_{j}}\right\} .
$$

After subtracting (3.1) from (3.2) and some simplification, one gets i).

ii) If one takes $z=i x$ with $0<x<1$ in i) and divides by $i$, one gets

$$
\begin{gathered}
\nu(3) x^{17}-\sum_{p \in \mathbf{M}} \nu(p) x^{2 p+1}+\sum_{p \in \mathbf{F}} \nu(p) x^{2 p-1}= \\
=-\sum_{d=3 ; \text { odd }}^{\infty} \mu(d) \frac{\nu(d)}{1+x^{2 d}}\left\{\sum_{j=1}^{2^{\omega(d)}} \chi\left(a_{j}\right) x^{a_{j}}\right\},
\end{gathered}
$$

a formula which is indeed true by analytic continuation for $|x|<1$.

iii) Recall that the Hadamard product of two functions $H(t)=\sum_{0}^{\infty} \alpha_{n} t^{n}, G(t)=$ $\sum_{0}^{\infty} \beta_{n} t^{n}$ is defined by $H(t) \otimes G(t)=\sum_{0}^{\infty} \alpha_{n} \beta_{n} t^{n}$ which is equal to $\frac{1}{2 \pi i} \int_{\gamma_{r}} H(q) G(t / q) \frac{d q}{q}$ using Cauchy formula, under suitable conditions for $t, r, \alpha_{n}, \beta_{n}$, which are met in our case. 
Now we take the Hadamard product of $G(z):=\sum_{3 \leq n o d d}^{\infty} z^{n^{2}-1}$ and $H(z):=$ $\sum_{m=1}^{\infty} h(m) z^{m}$ where

$$
\begin{gathered}
h(m):=\sum_{d \mid m} \mu(d) \log ^{2} d=\sum_{d \mid m} \mu(d)\left(\log \left(\frac{d}{m}\right)+\log m\right)^{2}= \\
\sum_{d \mid m} \mu(d)\left(2 \log \left(\frac{d}{m}\right) \log m+\log ^{2}\left(\frac{m}{d}\right)\right)=-2 \log m \Lambda(m)+\mu * \log ^{2}(m),
\end{gathered}
$$

and $*$ is the arithmetical convolution. From the last formula one sees that $\sum_{1}^{\infty} \frac{h(m)}{m^{s}}=$ $-2\left(\frac{\zeta^{\prime}(s)}{\zeta(s)}\right)^{\prime}+\frac{\zeta^{\prime \prime}(s)}{\zeta(s)}$.

Thus in this case

$$
\begin{gathered}
H(z) \bigotimes G(z)=\sum_{n=3 ; n \text { odd }}^{\infty} h\left(n^{2}-1\right) z^{n^{2}-1}= \\
-\log ^{2} 2 z^{8}+2 \log 2 \log 3 z^{288}+ \\
+2 \log 2 \sum_{p \in \mathbf{M}} \log p z^{(2 p+1)^{2}-1}+2 \log 2 \sum_{p \in \mathbf{F}} \log p z^{(2 p-1)^{2}-1},
\end{gathered}
$$

the last equality follows from Lemma $2($ with $\nu(d)=\log (d)$ ). After some slight simplification, iii) follows.

iv) Set $L_{\nu}(z):=\nu(3) z^{17}+\sum_{p \in \mathbf{M}} \nu(p) z^{2 p+1}+\sum_{p \in \mathbf{F}} \nu(p) z^{2 p-1}$. Then

$$
L_{\nu_{0}}(z)+L_{\nu_{1}}(z)=2 \sum_{3<p \in \mathbf{F}} z^{2 p-1}
$$

where the completely additive functions $\nu_{i}$ are defined for prime numbers as $\nu_{0}(p)=$ $1, \nu_{1}(p)=\chi(p)$. Now use (1.3) to get iv) of Theorem 1 .

Proof of Theorem 2: Multiply $(1.3)$ by $(1-z)$. If the number of Mersenne and Fermat primes were finite then the left hand side of (1.3) would tend to zero as $z \rightarrow 1-$. Thus, from the right hand side of (1.3) one would obtain (1.8). This finishes our proof.

\section{FinAl REMARKS.}

We write $f_{1}(z) \sim f_{2}(z)$ as $z \rightarrow 1-$ (meaning that both functions behave in a similar way as $z \rightarrow 1-)$ if $\lim _{z \rightarrow 1-} f_{1}(z)-f_{2}(z)=a$ for some real number $a$.

We make an observation of Theorem 1 (i) : is it true that, for some choice of $\nu$,

$$
\sum_{d=3 ; d \text { odd }}^{\infty} \mu(d) \frac{\nu(d)}{1-z^{2 d}}\left\{\sum_{j=1}^{2^{\omega(d)}} z^{a_{j}}\right\} \sim \sum_{d=3 ; d o d d}^{\infty} \mu(d) \frac{\nu(d)}{1-z^{2 d}}\left\{\left(2^{\omega(d)}-2\right) z^{d}+2 z^{2 d}\right\},
$$

as $z \rightarrow 1-?$ 
The reason for this is that, with the notation of Theorem 1 i) and using (1.7), the polynomial

$$
P_{2 d+1}(z):=\left\{\sum_{j=1}^{2^{\omega(d)}} z^{a_{j}}\right\}-\left\{\left(2^{\omega(d)}-2\right) z^{d}+2 z^{2 d}\right\},
$$

of degree $2 d+1$ has a double zero at $z=1$. Indeed, $P_{2 d+1}(z)=(z-1)^{2} z^{2 d-1}$ if $d$ is prime.

Set $g_{0}(z):=-\sum_{n \geq 3, n \text { odd }, n=p^{r}} z^{n}$. For $\nu(n)=\omega(n)$ the right hand side of formula (4.1) can be written in closed form. In this case, we prove below the following lemma.

Lemma 4. If $|z|<1$

$$
\begin{gathered}
\sum_{d=3 ; d o d d}^{\infty} \mu(d) \frac{\omega(d)}{1-z^{2 d}}\left\{\left(2^{\omega(d)}-2\right) z^{d}+2 z^{2 d}\right\}= \\
-2\left\{g_{0}(z)-g_{0}\left(z^{2}\right)-g_{0}\left(z^{4}\right)-g_{0}\left(z^{8}\right)-g_{0}\left(z^{16}\right)-\ldots\right\}+2 \sum_{n \geq 3 ; n \text { odd }} \omega(n)(-1)^{\omega(n)} z^{n} .
\end{gathered}
$$

Thus using Theorem 1 i) this raises the following

Question. Let $g_{0}(z)$ be defined as above. Is it true that

$$
\begin{gathered}
1+\sum_{p \in \mathbf{M}} z^{2 p+1}+\sum_{p \in \mathbf{F}} z^{2 p-1} \sim \\
2\left\{g_{0}(z)-g_{0}\left(z^{2}\right)-g_{0}\left(z^{4}\right)-g_{0}\left(z^{8}\right)-g_{0}\left(z^{16}\right)-\ldots\right\}+2\left\{\sum_{n \geq 3 ; n \text { odd }} \omega(n)(-1)^{\omega(n)+1} z^{n}\right\},
\end{gathered}
$$

as $z \rightarrow 1-$ ?

Proof of Lemma 4: Set $g(w):=\sum_{n=1, n o d d}^{\infty}\left(\sum_{d \mid n} b_{d}\right) w^{n}$. Then, in general,

$$
\begin{gathered}
\sum_{d \geq 1, d \text { odd }} b_{d} \frac{w^{d}}{1-w^{d}}=\sum_{n=1, n \text { odd }}^{\infty}\left(\sum_{d \mid n} b_{d}\right) w^{n}+\sum_{n=2, \text { neven }}^{\infty}\left(\sum_{d \mid n, d \text { odd }} b_{d}\right) w^{n}= \\
g(w)+g\left(w^{2}\right)+g\left(w^{4}\right)+g\left(w^{8}\right)+g\left(w^{16}\right)+\ldots
\end{gathered}
$$

and also

$$
\sum_{d \geq 1, d \text { odd }} b_{d} \frac{z^{d}}{1-z^{2 d}}=g(z) .
$$

Thus using these formulas, with $w=z^{2}$, and noticing that

$$
\sum_{d \mid n} \mu(d) \omega(d) 2^{\omega(d)}=2 \omega(n)(-1)^{\omega(n)} ; \sum_{d \mid n} \mu(d) \omega(d)=\left\{\begin{array}{l}
0, \text { if } n=1 \\
-1, \text { if } \omega(n)=1 \\
0, \text { if } \omega(n)>1
\end{array}\right.
$$

one sees that the right hand side of $(4.1)$, with $\nu(n)=\omega(n)$, is equal to 


$$
\begin{gathered}
-2 \sum_{d=3 ; d \text { odd }}^{\infty} \mu(d) \omega(d) \frac{z^{d}}{1-z^{2 d}}+2 \sum_{d=3 ; d \text { odd }}^{\infty} \mu(d) \omega(d) \frac{z^{2 d}}{1-z^{2 d}}+\sum_{d=3 ; d \text { odd }}^{\infty} \mu(d) \omega(d) 2^{\omega(d)} \frac{z^{d}}{1-z^{2 d}} \\
=-2 g_{0}(z)+2\left\{g_{0}\left(z^{2}\right)+g_{0}\left(z^{4}\right)+g_{0}\left(z^{8}\right)+\ldots\right\}+2 \sum_{n \geq 3 ; n \text { odd }} \omega(n)(-1)^{\omega(n)} z^{n},
\end{gathered}
$$

where $g_{0}$ is the function $g$ in case $b_{d}=\mu(d) \omega(d)$.

The referee suggested the following formula: let $g(n, m)$ be any complex or real function of the positive integers $n, m$ and $\nu(d)$ any completely additive function. If $m^{\prime}$ is square free then

$$
\begin{gathered}
\sum_{d \mid m^{\prime}} \mu(d) \nu(d)^{3} g(\omega(d), m)=\sum_{p \mid m^{\prime}} \nu(p)^{3} \sum_{r=0}^{\ell-1}(-1)^{r-1} g(r+1, m)\left(\begin{array}{c}
\ell-1 \\
r
\end{array}\right) \\
+3 \sum_{p q \mid m^{\prime}}\left(\nu(p)^{2} \nu(q)+\nu(q)^{2} \nu(p)\right) \sum_{r=0}^{\ell-2}(-1)^{r} g(r+2, m)\left(\begin{array}{c}
\ell-2 \\
r
\end{array}\right) \\
+6 \sum_{p q s \mid m^{\prime}} \nu(p) \nu(q) \nu(s) \sum_{r=0}^{\ell-3}(-1)^{r+1} g(r+3, m)\left(\begin{array}{c}
\ell-3 \\
r
\end{array}\right) .
\end{gathered}
$$

This identity may be proved along the lines of the proof of Lemma 1 using the identity $\left(\sum_{j=1}^{k} x_{j}\right)^{3}=\sum_{j=1}^{k} x_{j}^{3}+3 \sum_{1 \leq i<j \leq k}\left(x_{i}^{2} x_{j}+x_{j}^{2} x_{i}\right)+6 \sum_{1 \leq i<j<s \leq k} x_{i} x_{j} x_{s}$.

ACKNOWLEDGMENTS. We would like to thank the referee for carefully reading this note which helped to improve our presentation. We would like to express our deep gratitude to Professor Jeffrey C. Lagarias for his interest and kindness and also for suggesting several improvements in this note. We thank Professor Samuel Wagstaff Jr. for sending us his papers.

\section{REFERENCES}

[1] J. M. Borwein and P. B. Borwein, Pi and the AGM, Canadian Mathematical Society, Series of Monographs and Advanced Texts, 4, Wiley, New York, 1987.

[2] R. Crandall and C. Pomerance, Prime numbers, A computational perspective, Springer, New York, 2001.

[3] D. B. Gillies, Three new Mersenne primes and a statistical theory, Math. Comp., 18 (1964), 93-97.

[4] S. W. Golomb, The lambda method in prime number theory, J. Number Theory 2 (1970), 193-198.

[5] S. Ligh and L. Neal, A note on Mersenne numbers, Math. Mag. 47 (1974), 231-233.

[6] M. R. Schroeder, Where is the next Mersenne prime hiding?, Math. Intelligencer 5 (1983), no. $3,31-33$.

[7] E. C. Titchmarsh, The theory of the Riemann zeta-function, Second edition, Oxford Univ. Press, New York, (revised by D. R. Heath-Brown), 1986.

[8] S. S. Wagstaff, Jr., Prime numbers with a fixed number of one bits or zero bits in their binary representation, Experiment. Math. 10 (2001), no. 2, 267-273.

[9] S. S. Wagstaff, Jr., Divisors of Mersenne numbers, Math. Comp. 40 (1983), no. 161, 385-397. 
AMS Classification Numbers: 11A41. Key words and phrases. Fermat primes, Mersenne primes.

Departmento e Instituto de Matematica (INMABB), Universidad Nacional del Sur, Av. Alem 1253, Bahia Blanca, (8000), Argentina

E-mail address: ppanzone@uns.edu.ar 